\title{
ON THE ZEROS OF LINEAR PARTIAL FRACTIONS*
}

\author{
BY \\ MORRIS MARDEN $\dagger$ \\ I. INTRoduction
}

1. Problem and method. $\ddagger$ The present paper owes its origin to a suggestion from Professor Walsh $\S$ that I try to carry to a correct completion Professor Coble's analytical proof $\|$ of the following theorem due to the former:T

* Presented to the Society, December 27, 1928; received by the editors in January, 1929.

$\dagger$ National Research Fellow.

$\ddagger$ Bibliography. The following articles are cited at least twice. Each will hereafter be referred to by the letter $B$ and a roman numeral.

BI: M. Bocher, Proceedings of the American Academy of Arts and Sciences, vol. 40 (1904), pp. 469-484.

BII: J. L. Walsh, Comptes Rendus du Congrès International des Mathématiciens, Strasbourg, 1920 , pp. 1-4.

BIII: J. L. Walsh, these Transactions, vol. 19 (1918), pp. 291-298.

BIV: J. L. Walsh, these Transactions, vol. 22 (1921), pp. 101-116.

BV: J. L. Walsh, these Transactions, vol. 24 (1922), pp. 31-69.

BVI: J. L. Walsh, Proceedings of the National Academy of Sciences, vol. 8 (1922), pp. 139-141. BVII: J. L. Walsh, Rendiconti del Circolo Matematico di Palermo, vol. 46 (1922), pp. 1-13.

BVIII: A. B. Coble, Bulletin of the American Mathematical Society, vol. 27 (1921), pp. 434-437. BIX: M. Marden, Bulletin of the American Mathematical Society, vol. 35 (1929), pp. 363-370.

\& I wish to express my deep gratitude to Professor Walsh for his many suggestions and criticisms, and, above all, his constant encouragement in the course of this work.

\| This theorem was proved geometrically by Walsh (see BIV). Subsequently an analytical treatment of the same problem was published by Coble (see BVIII). Although suggestive as to the manipulation of the difficult algebra of the problem, the latter article is open to the following objections. (1) It quotes Walsh's theorem incorrectly by substituting "the interior of a circle" for "the circular region." (2) It considers in the proof only the case of the regions $C_{j}$ being the interiors of circles. (3) It implicitly assumes that the locus is finite whenever the regions $C_{j}$ are finite. This need not be the case, as is shown in chapter IV $\$ 2$ of the present paper. (4) It implicitly assumes without proof that the locus is a simply-connected region. Walsh's theorem, as stated by Coble, was later challenged by T. Nakahara (Tohoku Mathematical Journal, vol. 23 (1924), p. 97) as if it were Walsh's own statement. Nakahara's contribution to the subject consists of a set of simple conditions for the locus of the theorem to be finite. The conditions given are, however, only sufficient, not both necessary and sufficient, as announced by Nakahara. They may be deduced more easily by the methods of chapter IV $\$ 2$ of the present paper.

I See BIV, pp. 101-112. In Walsh's statement of the theorem, the point $z$ is defined by means of the real constant cross ratio $\left(z z_{1} z_{2} z_{3}\right)=\lambda$. Our definition is, however, equivalent to his, provided $\lambda \neq 0, \infty$.

By a circular region is meant in the theorem the interior and circumference of a circle, the exterior and circumference of a circle, a half-plane including its boundary line, or an entire plane. 
If the points $z_{1}, z_{2}, z_{3}$ vary independently and have circular regions as their respective loci, then the locus of the point $z$ defined by the equation

$$
\frac{m_{1}}{z-z_{1}}+\frac{m_{2}}{z-z_{2}}+\frac{m_{3}}{z-z_{3}}=0
$$

where $m_{1}, m_{2}, m_{3}$ are real constants whose sum is zero, is also a circular region.

In the course of my attempts in this direction, I found that the methods needed in the study of this problem were sufficiently powerful for an investigation of the zeros of the more general partial fraction

$$
F=\sum_{j=1}^{q+1} \frac{m_{j}}{z-z_{j}}
$$

where the $m_{j}$ are real constants whose sum is or is not zero. This investigation leads to a wide generalization, after the manner of Walsh, of theorems due to Gauss, Lucas, Bôcher and Walsh,* concerning the location of the zeros of the derivative of a rational function and of the zeros of the jacobian of two binary forms. For instance, its results enable one to locate approximately the positions of the zeros of the derivative of the polynomial

$$
f(z)=f_{1}(z) f_{2}(z) \cdots f_{k}(z)
$$

when all the zeros of each factor polynomial $f_{j}(z)$ are known to lie in or on a circle $C_{j}$. The approximation given is furthermore the best possible, in the sense that the geometrical limits involved are actually attained.

In place of merely proving the above theorem, I therefore set out to solve the following problem: To find the locus $R$ of the zeros of the partial fraction $F$ when the points $z_{j}$ vary independently and have circular regions $C_{i}$ as their respective loci. $\dagger$

The answer to this general problem turns out to be a reasonable generalization of the above theorem. In place of a circular region as the locus of the zeros of $F$ comes what we shall call a $p$-circular domain. $\ddagger$ By a $p$-circular domain we shall mean either the entire plane or a set of simply-connected

* See chapters III and V for statements of these theorems.

$\dagger$ Two interpretations of the problem are the following:

(a). Suppose a particle of mass $m_{j}$ is placed at each point $z_{j}$,-a particle which repels every other particle according to the inverse distance law. In the field of force so created, the zeros of $F$ are the points of equilibrium. Our problem is to find the whereabouts of these points of equilibrium, when it is known that each point $z_{j}$ may occupy any position within its circular region $C_{j}$. (Cf. BI, p. 475.)

(b). Given the function $z=f\left(z_{1}, z_{2}, \cdots, z_{q+1}\right)$ defined by the equation $F=0$ in a certain chunk of $(q+1)$-dimensional complex space. Our problem is to find the image of this chunk upon the z-plane.

$\ddagger$ C. $\mathrm{BV}$, footnote p. 39 , where Walsh guesses the result for the locus $R$, and BV, D. 38-43, where he derives some necessary properties of the locus $R$. 
closed regions which contain all the ovals of a given $p$-circular $2 p$-ic curve and which are bounded only by ovals of this $2 p$-ic curve.* For example, if $p$ were equal to two and the bicircular quartic consisted of one oval enclosed in a second, then as a bicircular domain we might have the entire plane, the interior and boundary of the outer oval, or the exterior and boundary of the inner oval, but not the ring-shaped region between the two ovals.

More specifiçally, we shall find the locus $R$ to be a $p$-circular domain, where

$$
p=q-1+\lim _{s \rightarrow 0}\left(\frac{n}{n+s},\right) \text { and } n=\sum_{j=1}^{q+1} m_{j} .
$$

This domain, if not the entire plane, is bounded by part or all of the $p$ circular $2 p$-ic curve with the equation $\dagger$

$$
\sum_{j=1}^{q+1} \frac{n m_{j}}{C_{j}}-\sum_{j=1, k=j+1}^{q+1} \frac{m_{j} m_{k} \Delta_{j k}}{C_{j} C_{k}}=0,
$$

where $C_{j}=0$ is the equation of the circle bounding the circular region $C_{j}$, and where $\Delta_{j k}$ is, according to a definite rule, the square of either the external or internal common tangent of the circles $C_{j}$ and $C_{k}$.

As our way to this result is rather long, it is perhaps worthwhile here to outline our method of attack and to point out its.principal difficulties.

We shall obtain the above information about the locus $R$ by successively determining certain two-dimensional envelopes. $f$ If $R_{k}$ denotes the locus of the zeros of $F$ when $z_{1}, z_{2}, z_{3}, \cdots, z_{k}$ vary over their respective circular regions but the points $z_{k+1}, z_{k+2}, \cdots, z_{q+1}$ are fixed, then $R_{k}$ may also be regarded as the envelope of the loci $R_{k-1}$ when $z_{k}$ describes as locus the circular region $C_{k}$. Thus, by computing successively the envelopes $R_{0}, R_{1}, \cdots$, $R_{q+1}$, we finally arrive at the locus $R$.

This method of successive envelopes entails as principal steps (1) the algebraic computation of the equations of the possible boundary curves of each locus $R_{k}$; (2) from these possible boundary curves the choice of the actual boundary curve of each locus $R_{k}$; and (3) the proof that each locus $R_{k}$ is composed only of simply-connected regions.

* We mean by a $p$-circular $2 p$-ic curve one which is represented by an equation of the form $a\left(x^{2}+y^{2}\right)^{p}+f(x, y)=0$

where $f(x, y)$ is a polynomial of degree less than $2 p$. If $a \neq 0$, the curve intersects the line at infinity (in the projective plane) $p$ times in each circular point at infinity and therefore cannot meet this line in any additional points. In other words, if $a \neq 0$, the curve consists entirely of closed ovals.

$\dagger$ The entire left-hand side of the equation given above lacks, of course, the factor $\prod_{j=1}^{q+1} C_{j}$. This factor will be omitted quite often in this paper, for the purpose of simplifying the formulas.

$\ddagger$ This method is suggested in the footnote of BIV, p. 102 . 
As a gradual approach to the difficulties involved, we shall consider at first two special cases: in chapter II, a case (case I) where all the $m_{j}$ are positive real numbers and the regions $C_{j}$ are finite, and in chapter IV, case II, where the $m_{j}$ are real numbers whose sum is zero, but where the regions $C_{i}$ are arbitrary. The advantage of this approach is that the work once done for case I in connection with steps (1) and (3) holds almost equally for case II and that therefore in case II we are left free to overcome the difficulties of step (2) caused by the arbitrariness of the numbers $m_{j}$ and the regions $C_{j}$. Furthermore, from the results of case II, we shall readily be able to infer the results in the most general case.

Having thus found the locus $R$, we shall try to verify our results as including those of Walsh and, finally in a closing chapter, we shall present two generalizations, the one for the zeros of the expression $F+\lambda$ where $\lambda$ is a constant, real or complex, and the other for the zeros of $F$ when the $m_{j}$ are complex constants.

2. Notation. We now proceed to introduce a system of abridged notation which will enable us expeditiously to perform the first of the above-described steps in the determination of the loci $R_{k}$. This system is essentially the same as that used by Coble in BVIII. Like him, we employ the symbol $\left[C_{1} C_{2}\right]$ for the invariant

$$
2\left(A_{1} A_{2}+B_{1} B_{2}\right)-b_{1} a_{2}-b_{2} a_{1} \equiv a_{1} a_{2}\left(R_{1}^{2}+R_{2}^{2}-d_{12}^{2}\right),
$$

which frequently appears in the computations. In the identity (1.21) $R_{i}$ is the radius of the circle $C_{j}, d_{12}$ is the distance between the centers of the circles $C_{1}$ and $C_{2}$ and the other letters are the coefficients in the equation

$$
C_{j} \equiv a_{j}\left(x^{2}+y^{2}\right)-2 A_{j} x-2 B_{j} y+b_{j}=0,
$$

$a_{j}, A_{j}, B_{j}, b_{j}$ all being real numbers. If $C_{j}$ is written in the form

$$
C_{j} \equiv a_{j} z \bar{z}-\bar{\beta}_{j} z-\beta_{j} \bar{z}+b_{j}=0,
$$

where $z=x+i y$ and $\beta_{j}=A_{j}+i B_{j}$, then

$$
\left[C_{1} C_{2}\right]=\beta_{1} \bar{\beta}_{2}+\bar{\beta}_{1} \beta_{2}-a_{1} b_{2}-a_{2} b_{1} .
$$

As may be easily verified, the quantity $\left[C_{1} C_{2}\right]$ obeys the following laws of combination:

$$
\begin{aligned}
{\left[C_{2} C_{1}\right] } & =\left[C_{1} C_{2}\right], \\
{\left[C_{1} k C_{2}\right] } & =k\left[C_{1} C_{2}\right] \\
{\left[C_{1} C_{2}+k\right] } & =\left[C_{1} C_{2}\right]-k a_{1}, \\
{\left[C_{1} C_{2}+C_{3}\right] } & =\left[C_{1} C_{2}\right]+\left[C_{1} C_{3}\right] \\
{\left[C_{1}+k C_{2} C_{1}+k C_{2}\right] } & =\left[C_{1} C_{1}\right]+2 k\left[C_{1} C_{2}\right]+k^{2}\left[C_{2} C_{2}\right]
\end{aligned}
$$$$
3
$$ 
where $k$ is an ordinary constant.

When $C_{i}$ degenerates into the null circle

$$
P_{j}=\left(x-x_{j}\right)^{2}+\left(y-y_{j}\right)^{2},
$$

additional laws are

$$
\begin{aligned}
& {\left[C_{j} P_{k}\right]=-C_{j k},} \\
& {\left[P_{k} P_{j}\right]=-P_{j k}=-P_{k j},}
\end{aligned}
$$

where $C_{j k}=C_{j}\left(z_{k}\right)$ and $P_{j k}=P_{j}\left(z_{k}\right)$. These latter quantities have the properties that, if the point $P_{k}$ is on the circle $C_{j}$, then $C_{j k}=0$; if $P_{k} \equiv P_{j}$, then $P_{j k}=0$; and always

$$
P_{j k}+P_{j l}-P_{k l} \equiv\left(z_{j}-z_{k}\right)\left(\bar{z}_{j}-\bar{z}_{l}\right)+\left(\bar{z}_{j}-\bar{z}_{k}\right)\left(z_{j}-z_{l}\right) .
$$

In terms of the above notation, the radius of the circle $C_{j}$ is $R_{j}{ }^{2}=$ $\left[C_{j} C_{j}\right] /\left(2 a_{j}^{2}\right)$ and the cosine of the angle between the two circles $C_{j}$ and $C_{k}$ is $\cos \left(C_{j}, C_{k}\right)=\left[C_{j} C_{k}\right] /\left\{\left[C_{j} C_{j}\right]\left[C_{k} C_{k}\right]\right\}^{1 / 2}$. As necessary and sufficient conditions we therefore have

(1) for $C_{j}$ to be a null circle: $\left[C_{j} C_{j}\right]=0$;

(2) for $C_{j}$ and $C_{k}$ to be orthogonal: $\left[C_{j} C_{k}\right]=0$; and

(3) for $C_{j}$ and $C_{k}$ to be mutually tangent:

$$
\left[C_{j} C_{k}\right]^{2}-\left[C_{j} C_{j}\right]\left[C_{k} C_{k}\right]=0 .
$$

The factors of the left hand side of (1.22), the invariants

and

$$
\begin{aligned}
\sigma_{j k} & \equiv \frac{1}{a_{j} a_{k}}\left\{\left[C_{j} C_{j}\right]^{1 / 2}\left[C_{k} C_{k}\right]^{1 / 2}-\left[C_{j} C_{k}\right]\right\} \\
& \equiv d_{j k}^{2}-\left(R_{j}-R_{k}\right)^{2}
\end{aligned}
$$

$$
\tau_{j k} \equiv-\frac{1}{a_{j} a_{k}}\left\{\left[C_{j} C_{j}\right]^{1 / 2}\left[C_{k} C_{k}\right]^{1 / 2}+\left[C_{j} C_{k}\right]\right\} \equiv d_{j k}^{2}-\left(R_{j}+R_{k}\right)^{2},
$$

are plainly, if positive, the squares of the external and internal common tangents respectively of the circles $C_{j}$ and $C_{k}$, and therefore must reduce to

$$
\sigma_{j k}=\tau_{j k}=C_{k j}
$$

when $C_{j} \equiv P_{j}$ and to

$$
\sigma_{j k}=\tau_{j k}=P_{j k}
$$

when both $C_{j} \equiv P_{j}$ and $C_{k} \equiv P_{h}$. 
II. Special case I: Positive numbers $m_{j}$. Finite circular Regions $C_{j}$

1. The locus $R_{0}$. The locus $R_{0}$ (cf. definition in I $\S 1$ ) consists merely of the $q$ zero-points of the partial fraction $F$. Since $n \neq 0$ and the points $z_{j}$ are finite, the points comprising $R_{0}$ are also finite.

The locus $R_{0}$ may be regarded as the null $q$-circular $2 q$-ic curve

$$
E_{0} \equiv\left\{\prod_{1}^{q+1}\left(z-z_{j}\right)\left(\bar{z}-\bar{z}_{j}\right)\right\}\left(\sum_{1}^{q+1} \frac{m_{j}}{z-z_{j}}\right)\left(\sum_{1}^{q+1} \frac{m_{j}}{\bar{z}-\bar{z}_{j}}\right)=0 .
$$

To write the latter expression entirely in terms of the notation of chapter I $\S 2$, we observe that, when $q=1$,

$$
\begin{aligned}
& \left(\frac{m_{1}}{z-z_{1}}+\frac{m_{2}}{z-z_{2}}\right)\left(\frac{m_{1}}{\bar{z}-\bar{z}_{1}}+\frac{m_{2}}{\bar{z}-\bar{z}_{2}}\right)=\frac{m_{1}^{2}}{P_{1}}+\frac{m_{2}^{2}}{P_{2}} \\
& \quad+\frac{m_{1} m_{2}}{P_{1} P_{2}}\left\{\left(z-z_{1}\right)\left(\bar{z}-\bar{z}_{2}\right)+\left(\bar{z}-\bar{z}_{1}\right)\left(z-z_{2}\right)\right\}=\frac{n m_{1}}{P_{1}}+\frac{n m_{2}}{P_{2}}-\frac{m_{1} m_{2} P_{12}}{P_{1} P_{2}} .
\end{aligned}
$$

This suggests what can be proved by mathematical induction; namely, that

$$
E_{0} \equiv\left(\prod_{1}^{q+1} P_{j}\right)\left\{\sum_{1}^{q+1} \frac{n m_{j}}{P_{j}}-\sum_{\substack{j=1 \\ k=j+1}}^{q+1} \frac{m_{j} m_{k} P_{j k}}{P_{j} P_{k}}\right\}
$$

2. The locus $R_{1}$. The locus $R_{1}$, evidently a bounded point set, may be considered as the map of the region $C_{1}$ (the locus of the point $z_{1}$ ) by means of the transformation $z=\psi\left(z_{1}\right)$ defined by the equation $F=0$. As the derivative

$$
\frac{d z}{d z_{1}}=\frac{m_{1}}{\left(z-z_{1}\right)^{2}}\left[\sum_{1}^{q+1} \cdot \frac{m_{j}}{\left(z-z_{j}\right)^{2}}\right]^{-1} \not \equiv 0, \infty,
$$

the transformation is conformal and consequently $R_{1}$ is a set of one or more bounded two-dimensional continua.

If $B_{1}$ denotes the boundary curve of $R_{1}$ and $z$ is any point of $B_{1}$, there must correspond to $z$ as a point of $R_{1}$ at least (actually only) one point $z_{1}$ in the region $C_{1}$. This point $z_{1}$ must lie on the circle $C_{1}$. For, were $z_{1}$ inside the circle $C_{1}$, a small neighborhood of $z_{1}$ could be mapped by the function $\psi\left(z_{1}\right)$ upon a neighborhood of $z$ consisting only of points of $R_{1}$. The point $z$ could under those circumstances not be a point of $B_{1}$, contrary to the hypothesis. Consequently, when $z_{1}$ traces the circle $C_{1}$, at least part of the resulting locus of the point $z$ is $B_{1}$.

The last fact enables us to obtain the equation of $B_{1}$ if we observe that, though representing in the variable $z$ a $q$-circular $2 q$-ic, the equation $E_{0}=0$ represents in the variable $z_{1}$ a null circle $G_{0}$. The null circle $G_{0}$ is, in fact, 
the point $z_{1}$ corresponding to a given point $z$. If $z$ is a point of $B_{1}$, by what has been proved above, $G_{0}$ lies on the circle $C_{1}$ and therefore*

$$
\left[E_{0} C_{1}\right]_{z_{1}}=0 \text {. }
$$

Computed according to the rules given in I $\S 2$, the left hand side of $(2.21)$ is

$$
E_{1} \equiv C_{1} \prod_{2}^{q+1} P_{j}\left\{\sum_{2}^{q+1} \frac{n m_{j}}{P_{j}}-\sum_{\substack{j=2 \\ k=j+1}}^{q+1} \frac{m_{j} m_{k} P_{j k}}{P_{j} P_{k}}\right\}+\prod_{2}^{q+1} P_{i}\left\{n m_{1}-\sum_{2}^{q+1} \frac{m_{1} m_{j} C_{1 j}}{P_{j}}\right\} .
$$

Every point $z$ of $B_{1}$ must therefore be a point of the curve $E_{1}=0$.

As is clear from the leading term of its equation, the curve $E_{1}=0$ is always a $q$-circular $2 q$-ic and as such consists exclusively of closed ovals. Among these ovals we may discern two types: the first, ovals $O_{1}$ which neither contain nor are contained in any other oval of $E_{1}=0$; the second, ovals $O_{2}$ not belonging to the first type.

The facts that $O_{1}$ forms part of $R_{1}$, that $R_{1}$ is a set of finite two-dimensional regions, and that $B_{1}$ is part of the curve $E_{1}=0$ certify to the entire interior of $O_{1}$ 's belonging to $R_{1}$. Whether or not the part of $R_{1}$ associated with an $O_{2}$ is simply-connected, needs however further investigation. For the present, we shall assume it also to be a simply-connected region (cf. II $\S 6)$.

The locus $R_{1}$ is therefore the interior and boundary of all the ovals of the $q$-circular $2 q$-ic curve $E_{1}=0$.

3. The locus $R_{2}$. Regarded as the envelope of the loci $R_{1}$, the locus $R_{2}$ consists also of a set of finite two-dimensional regions.

If $z$ is a point of $B_{2}$, the boundary curve of $R_{2}$, to $z$ corresponds (via the equation $F=0)$ at least one pair of points $\left(z_{1}, z_{2}\right)$ in the regions $C_{1}, C_{2}$ respectively. This pair must actually lie on the circles $C_{1}, C_{2}$. For, were, for example, $z_{1}$ inside the circle $C_{1}$, the point $z_{2}$ could be considered fixed and the arguments of II $\S 2$ used to contradict the hypothesis that the point $z$ is on $B_{2}$.

In other words, if the points $z_{1}$ and $z_{2}$ trace their respective circles simultaneously, the resulting locus of the point $z$ will include the curve $B_{2}$. That is to say, $B_{2}$ is part of the envelope of the curves $B_{1}$ or $E_{1}=0$ when the point $z_{2}$ describes as locus the circle $C_{2}$.

From this fact comes the equation of $B_{2}$ if we notice that, in the variable $z_{2}$, the equation $E_{1}=0$ represents a circle $G_{1}$. The circle $G_{1}$ corresponding to a point $z$ on $B_{2}$ must consist of at least one point on the circle $C_{2}$ and of no points

* The subscript $z_{1}$ means that in this operation $z_{1}$ is considered as the independent variable. 
inside the circle $C_{2}$; in other words, it must be tangent to $C_{2}$. A necessary condition on $z$ as a point of $B_{2}$ is therefore

$$
\left[E_{1} C_{2}\right]_{z_{3}}^{2}-\left[C_{2} C_{2}\right]_{z_{2}}\left[E_{1} E_{1}\right]_{z_{2}}=0 .
$$

Computed by the rules of I $\S 2$, the terms of the left hand side of this expression become

$$
\begin{aligned}
& -\left[E_{1} C_{2}\right]=\left(C_{1} C_{2} \prod_{3}^{q+1} P_{j}\right)\left\{\sum_{1}^{2} \frac{n m_{j}}{C_{j}}+\sum_{3}^{q+1} \frac{n m_{j}}{P_{j}}+\frac{m_{1} m_{2}\left[C_{1} C_{2}\right]}{C_{1} C_{2}}\right. \\
& \left.-\sum_{j=1, k=3}^{j=2, k=q+1} \frac{m_{j} m_{k} C_{j k}}{C_{j} P_{k}}-\sum_{j=3, k=j+1}^{q+1} \frac{m_{j} m_{k} P_{j k}}{P_{j} P_{k}}\right\} ; \\
& {\left[E_{1} E_{1}\right]=m_{1}^{2} m_{2}^{2}\left(\prod_{3}^{q+1} P_{j}\right)^{2}\left[C_{1} C_{1}\right] .}
\end{aligned}
$$

Equation (2.31) accordingly factors into the two equations

$$
\begin{aligned}
E_{2} & \equiv-\left[E_{1} C_{2}\right]-m_{1} m_{2} \prod_{3}^{q+1} P_{j}\left[C_{1} C_{1}\right]^{1 / 2}\left[C_{2} C_{2}\right]^{1 / 2}=0, \\
E_{2}^{\prime} & \equiv-\left[E_{1} C_{2}\right]+m_{1} m_{2} \prod_{3}^{q+1} P_{j}\left[C_{1} C_{1}\right]^{1 / 2}\left[C_{2} C_{2}\right]^{1 / 2}=0,
\end{aligned}
$$

at least one of which every point of $B_{2}$ must satisfy.

Both equations, having as leading term $n^{2}\left(x^{2}+y^{2}\right)^{q}$, represent $q$-circular $2 q$-ic curves. If $(\xi, \eta)$ is a point of the second, i.e. $E_{2}^{\prime}(\xi, \eta)=0$, then

$$
E_{2}(\xi, \eta)=-2 m_{1} m_{2}\left[C_{1} C_{1}\right]^{1 / 2}\left[C_{2} C_{2}\right]^{1 / 2} \prod_{3}^{q+1} P_{j}(\xi, \eta) \leqq 0 .
$$

The significance of the inequality (2.32) becomes clear if we observe that the continuous function $E_{2}(x, y)$ grows positively infinite as the point $(x, y)$ recedes indefinitely in any direction. The inequality (2.32) therefore expresses a sufficient condition for $(\xi, \eta)$ to lie inside or on an oval of $E_{2}=0$, i.e., for every oval of $E_{2}^{\prime}=0$ to be nested in an oval of $E_{2}=0$.

If now we assume (cf. II $\S 6$ ) that $R_{2}$ is also composed only of simplyconnected parts, we obtain $R_{2}$ as the interior and boundary of all the ovals of the $q$-circular $2 q$-ic

$$
\begin{aligned}
E_{2}= & \left(C_{1} C_{2} \prod_{3}^{q+1} P_{j}\right)\left\{\sum_{1}^{2} \frac{n m_{j}}{C_{j}}+\sum_{3}^{q+1} \frac{n m_{j}}{P_{j}}-\frac{m_{1} m_{2} \sigma_{12}}{C_{1} C_{2}}\right. \\
& \left.-\sum_{j=1, k=3}^{j=2, k=q+1} \frac{m_{j} m_{k} C_{j k}}{C_{j} P_{k}}-\sum_{j=3, k=j+1}^{q+1} \frac{m_{j} m_{k} P_{j k}}{P_{j} P_{k}}\right\}=0 .
\end{aligned}
$$


4. The locus $R_{N}$. We may now venture to suppose that $R_{N}$ consists of the interior and boundary of all the ovals of

$$
\begin{gathered}
E_{N}=\left(\prod_{1}^{N} C_{j} \prod_{N+1}^{q+1} P_{j}\right)\left\{\sum_{1}^{N} \frac{n m_{j}}{C_{j}}+\sum_{N+1}^{q+1} \frac{n m_{j}}{P_{j}}-\sum_{j=1, k=j+1}^{N} \frac{m_{j} m_{k} \sigma_{j k}}{C_{j} C_{k}}\right. \\
\left.-\sum_{j=1, k=N+1}^{j=q+1} \frac{m_{j} m_{k} C_{j k}}{C_{j} P_{k}}-\sum_{j=N+1, k=j+1}^{q+1} \frac{m_{j} m_{k} P_{j k}}{P_{j} P_{k}}\right\}=0 .
\end{gathered}
$$

On the principle of mathematical induction, let us test the correctness of this assumption by determining $R_{N+1}$.

Considerations similar to those of II $\S 3$ lead to the equation

$$
\left[E_{N} C_{N+1}\right]_{z_{N+1}}^{2}-\left[E_{N} E_{N}\right]_{z_{N+1}}\left[C_{N+1} C_{N+1}\right]_{z_{N+1}}=0
$$

as a necessary condition that a point $z$ lie on $B_{N+1}$, the boundary curve of $R_{N+1}$. Deciphered under the rules of I $\S 2$, the terms in (2.41) may be written as

$$
\begin{gathered}
-\left[E_{N} C_{N+1}\right]=\prod_{1}^{N+1} C_{j} \prod_{N+2}^{q+1} P_{j}\left\{\sum_{1}^{N+1} \frac{n m_{j}}{C_{j}}+\sum_{N+2}^{q+1} \frac{n m_{j}}{P_{j}}+\sum_{1}^{N} \frac{m_{N+1} m_{j}\left[C_{N+1} C_{j}\right]}{C_{N+1} C_{j}}\right. \\
\left.-\sum_{j=1, k=N+2}^{j=N+1, k=q+1} \frac{m_{j} m_{k} C_{j k}}{C_{j} P_{k}}-\sum_{j=N+2, k=j+1}^{q+1} \frac{m_{j} m_{k} P_{j k}}{P_{j} P_{k}}-\sum_{j=1, k=j+1}^{N} \frac{m_{j} m_{k} \sigma_{j k}}{C_{j} C_{k}}\right\}, \\
{\left[E_{N} E_{N}\right]=\left\{\prod_{1}^{N} C_{j} \prod_{N+2}^{q+1} P_{j} m_{N+1} \sum_{1}^{N} \frac{m_{j}\left[C_{j} C_{j}\right]^{1 / 2}}{C_{j}}\right\}^{2} .}
\end{gathered}
$$

Every point of $B_{N+1}$ must therefore lie on at least one of the two curves

$$
\begin{aligned}
E_{N+1} \equiv & -\left[E_{N} C_{N+1}\right]-\left[E_{N} E_{N}\right]^{1 / 2}\left[C_{N+1} C_{N+1}\right]^{1 / 2}=0 \\
\equiv & \prod_{1}^{N+1} C_{j} \prod_{N+2}^{q+1} P_{j}\left\{\sum_{1}^{N+1} \frac{n m_{j}}{C_{j}}+\sum_{N+2}^{q+1} \frac{n m_{j}}{P_{j}}-\sum_{j=1, k=j+2}^{N+1} \frac{m_{j} m_{k} \sigma_{j k}}{C_{j} C_{k}}\right. \\
& \left.-\sum_{j=N+1, k=q+1} \frac{m_{j} m_{k} C_{j k}}{C_{j} P_{k}}-\sum_{j=N+2, k=j+1}^{q+1} \frac{m_{j} m_{k} P_{j k}}{P_{j} P_{k}}\right\}=0 ; \\
E_{N+1}^{(N+1)} \equiv & -\left[E_{N} C_{N+1}\right]+\left[E_{N} E_{N}\right]^{1 / 2}\left[C_{N+1} C_{N+1}\right]^{1 / 2}=0 \\
\equiv & \prod_{1}^{N+1} C_{j} \prod_{N+2}^{q+1} P_{j}\left\{\sum_{1}^{N+1} \frac{n m_{j}}{C_{j}}+\sum_{N+2}^{q+1} \frac{n m_{j}}{P_{j}}-\sum_{j=1, k=j+1}^{N+1} \frac{m_{j} m_{k} \Delta_{j k}}{C_{j} C_{k}}\right. \\
& \left.-\sum_{j=1, k=N+2}^{q+1, k=q+1} \frac{m_{j} m_{k} C_{j k}}{C_{j} P_{k}}-\sum_{j=N+2, k=j+1}^{q+1} \frac{m_{j} m_{k} P_{j k}}{P_{j} P_{k}}\right\}=0,
\end{aligned}
$$

where $\Delta_{j k}=\tau_{j k}$ if $i$ or $j=N+1$, but $\Delta_{j k}=\sigma_{j k}$ otherwise.

Let us now bring into play the fact that $B_{N+1}$ must be independent of the 
order in which the $z_{j}, j=1,2, \cdots, N+1$, have begun to vary. If, for instance, the orders of $z_{x}$ and $z_{N+1}$ are interchanged, the condition upon $z$ as a point of $B_{N+1}$ must be revised so as to read that $z$ satisfies at least one of the two equations $E_{N+1}^{(\alpha)^{\prime}}=0$ and $E_{N+1}^{(\alpha)}=0$. These new equations may be derived from the old (2.42) by permuting the $z_{\kappa}$ and $z_{N+1}$. As the form $E_{N+1}$ is symmetric in the subscripts $j=1,2, \cdots, N+1, E_{N+1}^{(\kappa)} \equiv E_{N+1}$, but

$$
\begin{aligned}
& E_{N+1}^{(k)} \equiv \prod_{1}^{N+1} C_{j} \prod_{N+2}^{q+1} P_{j}\left\{\sum_{1}^{N+1} \frac{n m_{j}}{C_{j}}+\sum_{N+2}^{q+1} \frac{n m_{j}}{P_{j}}-\sum_{j=1, k=j+1}^{N+1} \frac{m_{j} m_{k} \Delta_{j k}}{C_{j} C_{k}}\right. \\
&\left.-\sum_{j=1, k=N+2}^{\sum_{j+1, k=q+1}} \frac{m_{j} m_{k} C_{j k}}{C_{j} P_{k}}-\sum_{j=N+2, k=j+1}^{q+1} \frac{m_{j} m_{k} P_{j k}}{P_{j} P_{k}}\right\}=0
\end{aligned}
$$

where $\Delta_{i j}=\tau_{i j}$ if $i$ or $j=\kappa$, but $\Delta_{i j}=\sigma_{i j}$ otherwise.

Let us assume that not all of curves $E_{N+1}^{(k)}=0$ have an arc in common. If $\zeta$ is a point on the curve $E_{N+1}^{(1)}=0$ and $\nu$ a sufficiently small arc of $E_{N+1}^{(1)}=0$ containing $\zeta$, there will exist at least one curve $E_{N+1}^{(x)}=0$ which does not contain $\nu$. Let us further suppose that $\zeta$ is not on the curve $E_{N+1}=0$. If $\zeta$ lies on $B_{N+1}$, it must satisfy at least one of the two equations in each of the sets

$$
E_{N+1}=0, \quad E_{N+1}^{(1)}=0 ; \quad \text { and } \quad \text { (2) } \quad E_{N+1}=0, \quad E_{N+1}^{(\kappa)}=0 .
$$

Yet $\zeta$ fails to satisfy either equation of set (2). It follows therefore from (1) that every point of $B_{N+1}$ is a point of the curve $E_{N+1}=0$.

Up to this point we have supposed not all the curves $E_{N+1}^{(k)}=0$ to have an arc in common. That such is the general state of affairs is evident from the distinctness in the forms $E_{N+1}^{(k)}$. In case, however, the $E_{N+1}^{(k)}=0$ all have an arc in common, we shall use the following reasoning. We shall consider the manifold $M$ consisting of all possible instances of special case $I$ and its submanifold $M^{\prime}$ composed of all instances for which all the curves $E_{N+1}^{(k)}=0$ have an arc in common. The manifold $M$ is of dimensions $3(N+1)$, the variables being the centers and radii of the $N+1$ circles $C_{j}$. If the dimensions of $M^{\prime}$ were less than $3(N+1)$, it would be possible to draw in $M$ a continuous arc which terminates in any chosen point of $M^{\prime}$ but does not pass through any other points of $M^{\prime}$. It would accordingly be possible to pass to the case where the curves $E_{N+1}^{(k)}=0$ have a common arc, continuously through cases where this is not so. As the equation $E_{N+1}$ has coefficients depending continuously upon those of the equations $C_{j}=0$, the curve $B_{N+1}$ would consist, even in the case of the curves $E_{N+1}^{(k)}=0$ having a common arc, only of ovals of the curve $E_{N+1}=0$.

We have then but to prove that the dimensions of $M^{\prime}$ are less than $3(N+1)$. As the curves $E_{N+1}^{(k)}=0$ all have a common arc, any point of this 
arc has coördinates satisfying equations

$$
\begin{aligned}
\Gamma^{(s, t)} \equiv & E_{N+1}^{(s)}-E_{N+1}^{(t)} \\
\equiv 2 & \left(\prod_{1}^{N+1} C_{j} \prod_{N+2}^{q+1} P_{j}\right)\left\{\frac{m_{t}\left[C_{t} C_{t}\right]^{1 / 2}}{C_{t}}-\frac{m_{s}\left[C_{s} C_{s}\right]^{1 / 2}}{C_{s}}\right\} \\
& \quad\left\{\sum_{1}^{N+1} \frac{m_{j}\left[C_{j} C_{j}\right]^{1 / 2}}{C_{j}}-\frac{m_{s}\left[C_{8} C_{s}\right]^{1 / 2}}{C_{s}}-\frac{m_{t}\left[C_{t} C_{t}\right]^{1 / 2}}{C_{t}}\right\}=0,
\end{aligned}
$$

where $s \neq t$ and $s, t=1,2, \cdots, N+1$. The common arc is thus an arc of a circle $Q$ with various distinct equations of the form $\sum \lambda_{j} C_{i}=0$. Equating the several expressions for the center and radius of $Q$, we obtain the equations

$$
\begin{gathered}
\frac{\sum_{1}^{N+1} \lambda_{j} \alpha_{j}}{\sum_{1}^{N+1} \lambda_{j}}=\frac{\sum_{1}^{N+1} \mu_{j} \alpha_{j}}{\sum_{1}^{N+1} \mu_{j}}, \\
\frac{\left[\sum_{1}^{N+1} \lambda_{j} C_{j}, \sum_{1}^{N+1} \lambda_{j} C_{j}\right]}{\left(\sum_{1}^{N+1} \lambda_{j}\right)^{2}}=\frac{\left[\sum_{1}^{N+1} \mu_{j} C_{j}, \sum_{1}^{N+1} \mu_{j} C_{j}\right]}{\left(\sum_{1}^{N+1} \mu_{j}\right)^{2}} .
\end{gathered}
$$

As we assumed $M^{\prime}$ to exist, these equations are compatible and define $M^{\prime}$ as a manifold of dimensions lower than $3(N+1)$.

In every case, therefore, $B_{N+1}$ is made up only of ovals of the curve $E_{N+1}=0$. If we assume the locus $R_{N+1}$ to consist of simply-connected parts, it follows that $R_{N+1}$ is the interior and boundary of all the ovals of the curve $E_{N+1}=0$, a result which certifies to the correctness of the assumption concerning the locus $R_{N}$.

5. The locus $R \equiv R_{q+1}$. Its properties. By setting $N=q+1$ we finally conclude that the locus $R$ is the interior and the boundary of all the ovals of the $q$-circular $2 q$-ic curve*

* The following theorem also results by a limiting process from the work of the preceding sections:

If $\alpha_{j}{ }^{\prime}(j=1,2, \cdots, p)$ are the zeros of the partial fraction

$$
\frac{m_{j}}{z-\alpha_{j}}
$$

and $\zeta_{m}$ and $\zeta_{M}$ respectively the smallest and largest zeros of the partial fraction

where $\beta_{j}=\Re\left(\alpha_{j}\right)$, then

$$
\sum_{j+1}^{q+1} \frac{m_{j}}{z-\beta_{j}}
$$

$$
\zeta_{m} \leqq \Re\left(\alpha_{j}{ }^{\prime}\right) \leqq \zeta_{M}
$$

This is Linfield's theorem for the case $k=1$. See reference BV, p. 54, and B. Z. Linfield, these Transactions, vol. 25 (1923), p. 257. 


$$
\sum_{1}^{q+1} \frac{n m_{j}}{C_{j}}-\sum_{j=1, k=j+1}^{q+1} \frac{m_{j} m_{k} \sigma_{j k}}{C_{j} C_{k}}=0 .
$$

As equation (2.51) represents a circular curve, it is of interest to locate the singular foci of this curve. Proceeding in the usual way, we find the circular line through the point $(u, v)$,

$$
y=i x+v-i u,
$$

to intersect curve (2.51) in points with abscissas which satisfy the equation

$$
2^{q} x^{q}\left\{\prod_{1}^{q+1}\left(w-\alpha_{j}\right) \sum_{1}^{q+1} \frac{1}{w-\alpha_{j}}\right\}+\text { terms of lower degree }=0,
$$

where $u+i v=w$ and $a_{j}+i b_{j}=\alpha_{j}$. Line (2.52) will then be asymptotic to curve (2.51), and the point $(u, v)$ a singular focus of curve (2.51) if and only if $(u, v)$ is such that

$$
\sum_{1}^{q+1} \frac{1}{w-\alpha_{j}}=0 .
$$

The singular foci are hence the zeros of $F$ for the $z_{j}=\alpha_{j}$; or, what amounts to the same, the points of equilibrium in a field of force created by a particle of mass $m_{j}$ at each $\alpha_{j}$ repelling according to the inverse distance law. (See I $\$ 1$, footnotes.)*

For further geometric characterization of the singular foci when the $\boldsymbol{m}_{j}$ are integers, we may appeal to the theorem recently proved by Linfield that "the roots of the derivative of the rational function $f(z)=\Pi_{1}^{n}\left(z-z_{j}\right)^{\mu_{i}}$ are the multiple roots of $f(z)$ to one lower order and the foci of a curve of class $n-1$ which touches each segment $z_{j} z_{k}(j \neq k=1,2, \cdots, n)$ at the points dividing it in the ratio $\mu_{j}$ to $\mu_{k} . "$ Our singular foci are therefore the ordinary foci of Linfield's curve. $\dagger$

From the form of the equation (2.51) we can deduce two more facts about the locus $R$.

First, as is obvious, any point through which pass at least three of the circles $C_{j}$ is a point of curve (2.51) and therefore a point of the locus $R$.

Secondly, the common points of any two of the regions $C_{j}$ belong to the locus $R$.

For suppose a point $P$ were to lie in just two of the regions $C_{j}$, for definiteness, $C_{1}$ and $C_{2}$. Entirely within the regions $C_{1}$ and $C_{2}$ let us draw respectively

* The singular foci are therefore points of the locus $R$. The bicircular quartic

$$
\left(x^{2}+y^{2}+37\right)^{2}=64\left(x^{2}+25\right)
$$

which has its singular foci at the points $( \pm 4,0)$, points outside of its solitary oval, is an example of a $p$-circular $2 p$-ic which, on account of the theorem, cannot serve as the boundary curve of a locus $R$.

$\dagger$ B. Z. Linfield, Bulletin of the American Mathematical Society, vol. 27 (1920), p. 17, and these Transactions, vol. 25 (1923), pp. 239-258. 
new circles $C_{1}^{\prime}$ and $C_{2}^{\prime}$ which pass through the point $P$. The point $P$ is now such that

$$
C_{1}^{\prime}(P)=C_{2}^{\prime}(P)=0
$$

and $C_{j}(P)>0$ for all $j \neq 1$ or 2 . The expression

$$
\begin{aligned}
E^{\prime} \equiv C_{1}^{\prime} C_{2}^{\prime} \prod_{3}^{q+1} C_{j}\left\{\sum_{1}^{2} \frac{n m_{j}}{C_{j}^{\prime}}+\sum_{3}^{q+1} \frac{n m_{j}}{C_{j}}-\frac{m_{1} m_{2} \sigma_{12}^{\prime}}{C_{1}^{\prime} C_{2}^{\prime}}\right. \\
\left.-\sum_{j=1, k=3}^{j=2, k=q+1} \frac{m_{j} m_{k} \sigma_{j k}^{\prime}}{C_{j}^{\prime} C_{k}}-\sum_{j=3, k=j+1}^{q+1} \frac{m_{j} m_{k} \sigma_{j k}}{C_{j} C_{k}}\right\}
\end{aligned}
$$

formed at $P$ has then the value

$$
E^{\prime}(P)=-m_{1} m_{2} \sigma_{12}^{\prime} C_{3}(P) C_{4}(P) \cdots C_{q+1}(P) .
$$

As neither one of the circles $C_{1}^{\prime}$ and $C_{2}^{\prime}$ is contained in the other, $\sigma_{12}^{\prime}>0$ and therefore $E^{\prime}(P)<0$. This means (cf. II $\S 3$ ) that $P$ lies within the ovals of the $q$-circular $2 q$-ic curve $E^{\prime}=0$ and accordingly is a point of the locus $R^{\prime}$, the locus $R$ in which the circles $C_{1}^{\prime}$ and $C_{2}^{\prime}$ respectively replace the circles $C_{1}$ and $C_{2}$. But, as $R^{\prime}$ is included in the $R$ corresponding to all the original circles $C_{j}$, the point $P$ is contained in $R$.

If $P$ were assumed to be a common point of just three of the regions $C_{j}$, for example, $C_{1}, C_{2}, C_{3}$, we could draw inside each of these three regions new circles $C_{1}^{\prime}, C_{2}^{\prime}, C_{3}^{\prime}$ which pass through the point $P$. The point $P$ would then be a point of $R^{\prime \prime}$, the locus $R$ in which the new circles $C^{\prime}$ replace the old, and therefore a point of the locus $R$ corresponding to all of the original circles $C_{j}$. Similar arguments hold for common points of four or more of the regions $C_{j}$.

6. Simple-connectivity. There remains for the completion of our study of special case I only to establish the fact that each locus $R_{k}$ is composed of simply-connected regions. This we shall do for $k=2$, a typical case. We draw in $R_{2}$ an arbitrary simple closed curve $K$ and show that, without ever intersecting $B_{2}$, the curve $K$ can be shrunk continuously to a point in $R_{2}$.

Considered as the envelope of the $R_{1}$, the locus $R_{2}$ consists of two overlapping parts: a part $S_{2}$ composed of the interior points of the $R_{1}$ corresponding to $z_{2}=\alpha_{2}$, and a part $T_{2}$ composed of the closed areas swept out by the moving curve $B_{1}$, i.e., composed exclusively of points through which passes at least one curve $B_{1}$.

The curve $K$ is thus composed (1) entirely of points in $S_{2},(2)$ entirely of points in $T_{2}$, or (3) partly of points in $T_{2}$ and partly of points not in $T_{2}$. If we assume the $R_{1}$ to be of simply-connected parts, there is nothing further to prove for curves $K$ of type (1). On the same assumption, it is possible 
so to deform a curve of type (3) continuously that it becomes a curve of type (2). The proof of the simple-connectivity therefore rests upon the behavior of curves $K$ of the second type.

Let $K$ then be a curve of the second type and $w$ an arbitrary point on it. Through $w$ as a point of $T_{2}$ passes at least one curve $B_{1}$, i.e., the equation

$$
E_{1}\left(w, z_{2}\right)=0
$$

has at least one solution $z_{2}$ in the region $C_{2}$ and therefore the circle $G_{1}$ given by (2.61) has at least one point in the region $C_{2}$. We shall select one of these points and label it $w_{2}$.

Let us mark the point $w=W$ on $K$, to which will correspond the circle $G_{1}=\Lambda_{1}$ and the point $w_{2}=W_{2}$ on $\Lambda_{1}$. Beginning at $w=W$, as $w$ traces $K$ continuously clockwise, the circle $G_{1}$ alters continuously its center and radius beginning with those of $\Lambda_{1}$. With $G_{1}$ we so shift $w_{2}$ as to cause it to trace a continuous curve $\Gamma_{2}$ which begins at $w_{2}=W_{2}$. When $w$ finally returns to $W$, $G_{1}$ becomes again $\Lambda_{1}$ but $w_{2}$ ends at a point $w_{2}=W_{2}^{\prime}$ on $\Lambda_{1}$, not necessarily the same as $W_{2}$. To make $\Gamma_{2}$ a closed curve, we add to it an $\operatorname{arc} \lambda_{2}=W_{2} W_{2}^{\prime}$ of circle $\Lambda_{1}$, an arc lying in the region $C_{2}$.

In the same way it is possible to associate with $K$ a curve $\Gamma_{1}$ in the region $C_{1}$. We consider again any point $w$ on $K$ and the curve $B_{1}$ through it. As $B_{1}$ is the envelope of the curves $E_{0}=0$ when $z_{1}$ traces the circle $C_{1}$, there passes through $w$ at least one curve $E_{0}=0$; i.e., the equation

$$
E_{0}\left(w, w_{2}, z_{1}\right)=0
$$

has at least one solution $z_{1}$ on the circle $C_{1}$ and therefore the null circle $G_{0}$ given by (2.62) lies on the circle $C_{1}$. We shall denote this $G_{0}$ by $w_{1}$; in particular, we shall label the points corresponding to $w=W$ and $w_{2}=W_{2}$, and $w=W$ and $w_{2}=W_{2}^{\prime}$ by $w_{1}=W_{1}$ and $w_{1}=W_{1}^{\prime}$ respectively.

As the point $w$ and the corresponding point $w_{2}$ trace $K$ and $\Gamma_{2}$ from $W$ and $W_{2}$ to $W$ and $W_{2}^{\prime}$, the point $w_{1}$ describes on the circle $C_{1}$ a curve $\Gamma_{1}$ from $W_{1}$ to $W_{1}^{\prime}$. If the point $w$ is now held fast at $W$ and the point $w_{2}$ traces $\lambda_{2}$ from $W_{2}^{\prime}$ to $W_{2}$, the point $w_{1}$ traces an $\operatorname{arc} \lambda_{1}$ of the circle $C_{1}$, which arc closes $\Gamma_{1}$.

So far we have been concerned with selecting, from the infinitude of pairs $\left(z_{1}, z_{2}\right)$ leading to a point $w$ on $K$, just those which together form closed curves $\Gamma_{1}$ and $\Gamma_{2}$ in the regions $C_{1}$ and $C_{2}$. The correspondence thus set up between points $w$ of $K$ and $w_{1}$ and $w_{2}$ of $\Gamma_{1}$ and $\Gamma_{2}$ is one-to-one except that to the point $W$ corresponds all the points on the arcs $\lambda_{1}$ and $\lambda_{2}$. The correspondence between points $w_{1}$ of $\Gamma_{1}$ and $w_{2}$ of $\Gamma_{2}$ is one-to-one without exception. 
From now on we shall consider $K$ as generated by the point $z=w$ when $w_{2}$ and its corresponding $w_{1}$ trace out $\Gamma_{2}$ and $\Gamma_{1}$ simultaneously. By suitably deforming $\Gamma_{2}$ and $\Gamma_{1}$, we shall try to shrink $K$ to a point in $R_{2}$.

Continuous deformations of $\Gamma_{1}$ and $\Gamma_{2}$ subject to the following conditions will preserve the essential features of the above-formed correspondences between $w, w_{1}$ and $w_{2}$ : (1) the points $W_{1}$ and $W_{2}$ are to remain stationary; (2) points $w_{2}$ on $\lambda_{2}$ are to go into points $w_{2}^{\prime}$ also on $\lambda_{2}$, and the corresponding point $w_{1}$ into the null circle $E_{0}\left(W, w_{2}^{\prime}, w_{1}\right)=0$; and, finally, (3) the curves $\Gamma_{1}$ and $\Gamma_{2}$ are always to be closed curves lying in the regions $C_{1}$ and $C_{2}$.

Subject to these restrictions, let us modify the shapes of $\Gamma_{1}$ and $\Gamma_{2}$ slightly, causing $w_{1}$ and $w_{2}$ to go into such points $w_{1}^{\prime}$ and $w_{2}^{\prime}$ of $\Gamma_{1}^{\prime}$ and $\Gamma_{8}^{\prime}$ that

$$
\left|w_{2}-w_{2}^{\prime}\right|<\delta,\left|w_{1}-w_{1}^{\prime}\right|<\delta .
$$

A continuous function of $w_{1}$ and $w_{2}, w$ is carried into $w^{\prime}$ where

$$
\left|w-w^{\prime}\right|<\epsilon \text {, }
$$

and $\epsilon$ is a positive number arbitrarily small for sufficiently small $\delta$.

Of what points the curve $K^{\prime}$, the transform of $K$, consists may be seen if the point $w_{2}^{\prime}$ and the corresponding point $w_{1}^{\prime}$ are allowed to trace continuously complete circuits on $\Gamma_{2}^{\prime}$ and $\Gamma_{1}^{\prime}$, beginning at the points $w_{2}^{\prime}=W_{2}$ and $w_{1}^{\prime}=W_{1}$. The curve $K^{\prime}$ will be a continuous curve with $W$ and $W^{\prime}$ as terminal points, $W^{\prime}$ being another root of the equation $F=0$ where $z_{1}=W_{1}$ and $z_{2}=W_{2}$.

If $W \neq W^{\prime}$, the equation $F=0$ then has $r \geqq 2$ distinct roots: $Z_{1}=W$, $Z_{2}=W^{\prime}, Z_{3}, \cdots, Z_{r}$. For these a number $g\left(W_{1}, W_{2}\right)$ always exists so that

in particular,

$$
\left|Z_{i}-Z_{j}\right| \geqq g>0, i \neq j \text {; }
$$

$$
\left|W-W^{\prime}\right| \geqq g \text {. }
$$

On the other hand, $W^{\prime}$ may be thought of as the point $w^{\prime}$ into which $W$ has gone as result of the above-described deformations. From inequality (2.63) it then follows that

$$
\left|W-W^{\prime}\right|<\epsilon .
$$

For $\delta$ sufficiently small, we may take $\epsilon=g / 2$ causing inequalities (2.64) and (2.65) to contradict one another. Consequently, $W=W^{\prime}$ : the new $\dot{K}^{\prime}$ is a closed curve.

As total effect of our deformations on $\Gamma_{1}$ and $\Gamma_{2}$, the curve $K$ has therefore changed into another continuous closed curve $K^{\prime}$ in $R_{2}$ which passes through the point $W$. If now we contract $\Gamma_{1}$ and $\Gamma_{2}$ continuously to the 
points $W_{1}$ and $W_{2}$, the curve $K$ will, without ever cutting $B_{2}$, shrink continuously to the point $W$, a point in $R_{2}$.

In a similar way we may prove that all the other loci $R_{k}$ are composed of simply-connected parts. The curve $\Gamma_{k}$ is chosen as a closed curve entirely in the region $C_{k}$, but the other auxiliary curves $\Gamma_{k-p}$ as closed curves traced on the circles $C_{k-p}$ by the points of tangency of certain circles $G_{k-p-1}$.

\section{Applicatrons}

1. The derivative of a polynomial. The results of the previous chapter will now be applied to the location of the zeros of the derivative of the polynomial:

$$
f(z)=\prod_{1}^{n}\left(z-z_{j}\right)=\prod_{1}^{q+1} f_{j}(z),
$$

where $f_{j}$ is a polynomial of degree $m_{j}$. As

$$
f^{\prime}(z)=f(z)\left\{\sum_{1}^{n} \frac{1}{z-z_{j}}\right\},
$$

the zeros of $f^{\prime}(z)$ are the zeros of the partial fraction bracketed in the equation (3.1) and the multiple zeros of $f(z)$ to one lower order.*

Thus the locus of the zeros of $f^{\prime}(z)$ when all the zeros of each $f_{i}$ vary within and on a circle $C_{j}$ will be composed of two parts: a part $S$ due to the zeros of the partial fraction in (3.1) and a part $T$ due to the multiple zeros of $f(z)$. The former part $S$ has already been determined. The latter part $T$ consists of every point of any region $C_{j}$ for which $m_{j} \neq 1$ and the common points of any two of the regions $C_{j}$, for, at such a point, two or more points $z_{j}$ can be made to coincide. But, since the common points of any two regions $C_{j}$ are already included in the part $S$ (cf. II $\S 5$ ), we need not mention them again.

In short, then, if $f_{j}(z)$ and $f(z)=\Pi_{1}^{q+1} f_{j}$ are polynomials of degrees $m_{j}$ and $n$ respectively, and if all the zeros of each $f_{j}$ have as common locus the interior and circumference of a circle $C_{j}$, the locus of the zeros of $f^{\prime}(z)$ will consist of (a) the whole of every region $C_{j}$ for which $m_{j} \neq 1$ and $(\mathrm{b})$, when $q \geqq 1$, the locus $R$ of II $\S 5$.

Reasoning based on the continuity of the zeros, similar to that used by Bôcher and Walsh, BI, p. 478 and BIV, p. 113, may now be employed to prove at once that there will exist exactly $m_{j}-1$ zeros of $f^{\prime}(z)$ in the region

* This is essentially Gauss' theorem, Werke, vol. 3, 1816, p. 112, that the zeros of $f^{\prime}(z)$ are at the multiple zeros of $f(z)$ and at the points of equilibrium in the field of force created by unit particles at each $z_{j}$ repelling one another according to the inverse-distance law. 
$C_{i}$ and $q$ in (b) if no two of the regions $C_{i}$ overlap and if (b) does not have any points in common with any region $C_{i}$; and precisely one in each part of (b) if, furthermore, (b) breaks up into $q$ distinct parts.

2. Special cases. As a generalization, the theorem of the preceding section should reduce for special values of $q$ and special choices of the regions $C_{i}$ to existing theorems on the subject. Let us see whether this is so.

When $q=0$, the locus $R$, according to the theorem, is merely the region $C_{1}$; that is, the zeros of $f^{\prime}(z)$ lie in or on any circle enclosing the zeros of $f(z)$. This is but another way of expressing the Lucas theorem* that the zeros of $f^{\prime}(z)$ lie within any convex polygon enclosing the zeros of $f(z)$.

When $q=1$, our theorem accords with one proved originally by Walsh (BII, p. 1): the locus consists of the circular regions $C_{1}$ (if $m_{1} \neq 1$ ) and $C_{2}$ (if $m_{2} \neq 1$ ), and of the interior and circumference of the circle

$$
n m_{1} C_{2}+n m_{2} C_{1}-m_{1} m_{2} \sigma_{12}=0,
$$

with radius $r=\left(m_{2} r_{1}+m_{1} r_{2}\right) /\left(m_{1}+m_{2}\right)$ and center $\alpha=\left(m_{2} \alpha_{1}+m_{1} \alpha_{2}\right) /\left(m_{1}+m_{2}\right)$.

When $q=2$, our theorem again agrees with one proved by Walsh (BVI): the locus consists of the whole of every region $C_{j}$ for which $m_{j} \neq 1$ and the interior and boundary of all the ovals of the bicircular quartic (2.51).

3 . Special cases continued. If $q$ is taken as arbitrary, but all the circles $C_{j}$ are given a common external center of similitude, straightforward computation $\dagger$ proves the curve (2.51) to break up into the circles $C_{k}^{\prime}$ which have the same external center of similitude and the same common tangents as the circles $C_{j}$. The singular foci, according to their definition, are in this case the centers of the circles $C_{k}{ }^{\prime}$. All this agrees with a theorem due to Walsh (BV, p. 52).

Finally let us study the special case in which all the $m_{j}$ and all the circles $C_{j}$ are equal, and in which the centers of the circles $C_{j}$ lie at the vertices $V_{i}$ of a regular polygon of $q+1$ sides. Clearly the locus must be symmetric in the lines joining the center $O$ of the polygon with the points $V_{j}$.

If curve (2.51) does not degenerate into a set of circles with center at $O$, we may choose on curve (2.51) a point $P$ which is neither on any of the lines $O V_{j}$ nor on their angular bisectors. Because of the symmetry, the circle with center at $O$ and radius $O P$ would intersect the curve $(2.51)$ in $2(q+1)$ distinct points, i.e., twice more often than permissible.

* F. Lucas, Comptes Rendus, 1868, p. 469, and 1888, p. 121.

$\dagger$ I have carried out the details of this computation only for the case that the external center of similitude is at the point at infinity. For $q=2, \mathrm{I}$ have also been able to show our bicircular quartic coincident with Walsh's two circles by assuming the contrary and finding that it would then intersect these two circles in a total of more than sixteen points. I hope through further study of the properties of $p$-circular $2 p$-ic curves to be able to extend this geometrical method to the general case. 
Hence the locus is composed of the circular regions $C_{i}$ if $m_{j} \neq 1$ and of the interior and circumference of the circle with center at $O$ and with a radius equal to the largest real root of the equation

$$
\sum_{0}^{q} \frac{3}{t_{j}}-\sum_{j=0, k=i+1}^{q} \frac{\left\{2 h \sin \frac{\pi}{q+1}(j-k)\right\}^{2}}{t_{j} t_{k}}=0,
$$

where

$$
t_{i}=r^{2}+h^{2}-a^{2}-2 h r \cos \frac{2 \pi j}{q+1},
$$

and where $a$ is the radius of the circle $C_{i}$ and $h$ that of the circumscribing circle of the polygon.

When $q=2$, equation (3.31) becomes

$$
\left(r^{2}-a^{2}\right)\left(r^{2}+h^{2}-a^{2}\right)=h^{2},
$$

which has as largest root $r=\left(a^{2}+h a\right)^{1 / 2}$, a number also given by Walsh (BVI, p. 140).

When $q=3$, equation (3.31) reduces to

$$
\left(r^{2}-a^{2}\right)^{3}-2 h^{2} a^{2}\left(r^{2}-a^{2}\right)-h^{4} a^{2}=0,
$$

which, if $h=2^{1 / 2}$, has as largest root a number $T$,

$$
\left(a^{2}+2 a\right)^{1 / 2}<T \leqq\left(a^{2}+s a\right)^{1 / 2},
$$

where $s>2$ and is such that

$$
\frac{4}{s^{3}-4 s} \leqq a .
$$

Equality occurs in (3.32) when and only when it takes place in (3.33).

IV. Special case II: Numbers $m_{i}$ Summing to zero. Arbitrary CIRCULAR REGIONS $C_{i}$

1. Preliminary remarks. We now turn to the special case of real arbitrary $m_{j}$ 's summing to zero.

The zeros of $F$ have in this case the striking property of being invariant under linear transformation of the plane or stereographic projection upon the sphere. If a linear transformation takes the $z_{j}$ into the points $z_{j}^{\prime}$ the zeros of $F$ pass into the zeros of $F\left(z_{j}^{\prime}\right)$. If a stereographic projection throws the $z_{j}$ into the points $w_{j}$ on the sphere, the $q-1$ zeros of $F$ go into the $q-1$ positions of equilibrium on the sphere in the field of force due to particles 
of mass $m_{j}$ at the $w_{j}$ repelling according to the inverse-distance law. (Cf. BI, p. $469 \mathrm{ff}$.) If, then, we project the regions comprising the locus $R$ upon the sphere, the arguments of II $\S 6$ will serve to show that the images of these regions upon the sphere are simply-connected.

From the formal work of the second chapter, we infer at once that, when not the entire plane, the locus $R$ in case II is bounded by ovals of the curve

$$
\sum_{j=1, k=j+1}^{q+1} \frac{m_{j} m_{k} \Delta_{j k}}{C_{j} C_{k}}=0 .
$$

A new choice of $\Delta_{j k}$ between $\sigma_{j k}$ and $\tau_{j k}$ has however to be made in special case II for several reasons. No longer are the previous choices generally valid, because the $m_{j}$ and the leading coefficient of the left-hand side of equation (4.1) may now be either positive or negative, and because the regions $C_{i}$ and the locus $R$ may be finite or not finite.

To pass gently from chapter two, let us first make the new choice for the subcase in which both all the regions $C_{j}$ and the locus $R$ are finite. From this subcase we shall then proceed to the general situation by causing the regions $C_{i}$ of the subcase to expand continuously until they approach the $C_{i}$ of the general case.

But, before we may discuss this subcase, we need to obtain criteria for determining whether or not a given instance of finite regions $C_{i}$ will yield a finite locus $R$.

2. Criteria for a finite $R$. That finiteness in the regions $C_{j}$ does not ensure a finite locus $R$ is evident from the example

$$
\frac{1}{z-1}+\frac{1}{z+1}-\frac{2}{z}
$$

which has $z=\infty$ as its only zero, the regions $C_{i}$ being the points $z=0$ and $z= \pm 1$. (Contrast with BVIII.)

To obtain a condition for a finite $R$, let us express the equation $F=0$ in the form

$$
z^{q-1}\left\{\sum_{1}^{q+1} m_{j} z_{j}\right\}+\text { terms of lower degree }=0 .
$$

It is thus necessary and sufficient for $z=\infty$ to be in $R$ that there exist in each region $C_{i}$ a point $z_{j}$ making

$$
\sum_{1}^{q+1} m_{j} z_{j}=0
$$

In order to give this condition a more practical expression, let us determine the locus $L_{12}$ of the point $z_{q+1}$ defined by (4.22) when $z_{1}$ and $z_{2}$ vary respectively 
over the regions $C_{1}$ and $C_{2}$. Applying the method of $I \S 2$, we find here $\boldsymbol{R}_{0}$ to be the null circle

$$
e_{0}=-\left(\sum_{1}^{q+1} m_{j} z_{j}\right)\left(\sum_{1}^{q+1} m_{j} \bar{z}_{j}\right)=\sum_{j=1, k=j+1}^{q+1} m_{j} m_{k} P_{j k}=0,
$$

and $R_{2}$ or $L_{12}$ finally to be the interior and circumference of the circle

$$
D_{12}=m_{1} m_{2} \Delta_{12}+\sum_{j=1, k=3}^{j=2, k=q+1} m_{j} m_{k} C_{j k}+\sum_{j=3, k=j+1}^{q+1} m_{j} m_{k} P_{j k}=0
$$

with center at

As the radius of $D_{12}$ is

$$
-\frac{1}{m_{q+1}}\left\{\sum_{1}^{2} m_{j} \alpha_{j}+\sum_{3}^{q} m_{j} z_{j}\right\} .
$$

$$
\left|\frac{m_{1} r_{1}+m_{2} r_{2}}{m_{q+1}}\right| \text { if } \quad \Delta_{12}=\sigma_{12}
$$

and

$$
\left|\frac{m_{1} r_{1}-m_{2} r_{2}}{m_{q+1}}\right| \text { if } \quad \Delta_{12}=\tau_{12},
$$

the quantity $\Delta_{12}$ must be chosen equal to $\sigma_{12}$ if $m_{1} m_{2}>0$, and to $\tau_{12}$ if. $m_{1} m_{2}<0$.

When and only when this locus $L_{12}$ overlaps the circular region $C_{q+1}$ will condition (4.22) be fulfilled and the point $z=\infty$ belong to the locus $R$. In other words, for the locus $R$ to be finite, it is necessary and sufficient that every point $z_{q+1}$ of the region $C_{q+1}$ satisfy the inequality $D_{12}\left(z_{q+1}\right)<0$.

3. The finite subcase. Let us now determine the quantity $\Delta_{j k}$ in equation (4.1) for the subcase of finite regions $C_{i}$ and a finite locus $R$.

From the work of the second chapter, it is plain that to determine for instance $\Delta_{12}$, it is only necessary to specialize in (4.1) all the $C_{j}$ except $C_{1}$ and $C_{2}$ to be null circles, and to choose between the two equations

$$
\begin{aligned}
& E_{2}=C_{1} C_{2} \prod_{3}^{q+1} P_{j}\left\{\frac{m_{1} m_{2} \sigma_{12}}{C_{1} C_{2}}+\sum_{j=1, k=3}^{j=2, k=q+1} \frac{m_{j} m_{k} C_{j k}}{C_{j} P_{k}}+\sum_{j=3, k=j+1}^{q+1} \frac{m_{j} m_{k} P_{j k}}{P_{j} P_{k}}\right\}=0, \\
& E_{2}^{\prime}=C_{1} C_{2} \prod_{3}^{q+1} P_{i}\left\{\frac{m_{1} m_{2} \tau_{12}}{C_{1} C_{2}}+\sum_{j=1, k=3}^{j=2, k=q+1} \frac{m_{j} m_{k} C_{j k}}{C_{j} P_{k}}+\sum_{j=3, k=j+1}^{q+1} \frac{m_{j} m_{k} P_{j k}}{P_{j} P_{k}}\right\}=0 .
\end{aligned}
$$

The leading coefficient of the first is precisely $D_{12}$ with $\Delta_{12}=\sigma_{12}$ and that of the second, $D_{12}$ with $\Delta_{12}=\tau_{12}$ (cf. IV $\S 2$ ).

If $m_{1} m_{2}>0$ and $\zeta$ is any point on $E_{2}^{\prime}=0$, i.e., $E_{2}^{\prime}(\zeta)=0$, then

$$
E_{2}(\zeta)=2 m_{1} m_{2}\left[C_{1} C_{1}\right]^{1 / 2}\left[C_{2} C_{2}\right]^{1 / 2} \prod_{3}^{q+1} P_{j}(\zeta) \geqq 0
$$


Since $D_{12}<0$, inequality (4.31) signifies that all the ovals of the curve $E_{2}^{\prime}=0$ are contained in those of the curve $E_{2}=0$. Similarly, if $m_{1} m_{2}<0$, all the ovals of the curve $E_{2}=0$ are enclosed in ovals of the curve $E_{2}^{\prime}=0$.

The choice for $\Delta_{12}$ is typical. The locus $R$ consists of the interior and boundary of all the ovals of the $(q-1)$-circular $2(q-1)$-ic curve

$$
\sum_{j=1, k=j+1}^{q+1} \frac{m_{j} m_{k} \Delta_{j k}}{C_{j} C_{k}}=0,
$$

where $\Delta_{j k}=\sigma_{j k}$ if $m_{j} m_{k}>0$, and $\Delta_{j k}=\tau_{j k}$ if $m_{j} m_{k}<0$.

4. The general case. For convenience in our treatment of the unrestricted instance of special case II, let us introduce the notion of the "radius of a circular region C." Considering that direction positive along radii vectores which points into the region $C$, we define as the radius of the region $C$ ( $\tilde{r}$ where $r$ is the radius of circle $C$ ) the algebraic distance from the circumference to the center of $C$. Thus if the region $C$ were the exterior of the circle $C$, we should find $\tilde{r}<0$.

On each circle $C_{i}$ let us now choose a point $z_{i}^{*}$ such that

$$
\sum_{1}^{q+1} m_{j} z_{j}^{*} \neq 0,
$$

and construct within the region $C_{j}$ a circle $\gamma_{i}$, of radius $\omega_{j}$, tangent to the circle $C_{j}$ at $z_{j}^{*}$. Because of condition (4.22) the locus of the zeros of $F$ when the $z_{j}$ vary over the regions $\gamma_{j}$ (the interior and circumference of the circle $\gamma_{j}$ ) will be finite when the radii $\omega_{j}$ are sufficiently small. It will be in fact bounded by curve (4.32) where the $C_{j}$ are replaced by the $\gamma_{j}$.

If we allow the $\omega_{i}$ gradually to increase, the coefficients of (4.32) will change continuously. If $\tilde{r}_{p}<0$, the stereographic projection of the circle $\gamma_{p}$ upon the sphere must experience two stages in order to coincide with the circle $C_{p}$ : first the circle $\gamma_{p}$ must expand until it passes through the north pole of the sphere, i.e., $\tilde{\omega}_{p}$ must grow positively infinite; then it must continue to expand, thereafter the region $\gamma_{p}$ enclosing the north pole. That is to say, the quantity $\tilde{\omega}_{p}$ must increase from $-\infty$ to $\tilde{r}_{p}$.

In equation (4.32), accordingly, the forms only of

and

$$
\sigma_{j k}=d_{j k}^{2}-\left(r_{j}-r_{k}\right)^{2}
$$

$$
\tau_{j k}=d_{j k}^{2}-\left(r_{j}+r_{k}\right)^{2}
$$

are affected by the continuous expansions of the regions $\gamma_{j}$. If $\tilde{r}_{j} \tilde{r}_{k}<0$, $\sigma_{j k}$ and $\tau_{j k}$ are interchanged; otherwise, they too retain their original forms. In other words, provided, as we have implicitly assumed, the locus $R$ 
is not the entire plane, it consists of a set of simply-connected regions bounded by ovals of the $(q-1)$-circular $2(q-1)$-ic curve

$$
\sum_{j=1, k=j+1}^{q+1} \frac{m_{j} m_{k} \Delta_{j k}}{C_{j} C_{k}}=0
$$

where $\Delta_{j k}=\sigma_{j k}$ if $\left(m_{j} \tilde{r}_{j}\right)\left(m_{k} \tilde{r}_{k}\right)>0$ and $\Delta_{i k}=\tau_{j k}$ if $\left(m_{j} \tilde{r}_{j}\right)\left(m_{k} \tilde{r}_{k}\right)<0$. In short, the locus $R$ is always $a(q-1)$-circular domain.

To be noticed also is the type of curve which bounds the locus $R$. Since the quantities $\Delta_{j k}$ are invariants and the $C_{j}(x, y)$ covariants of the forms $C_{j}\left(x_{i}, y_{j}\right)$, the curve (4.42) is also a covariant of these forms. This is to be expected from the remarks of IV $\S 1$.

The locus $R$, just determined, may or may not include the point at infinity. Conditions that it should contain this point have already been found (IV $\S 2$ ) in the case that all the $\tilde{r}_{j}$ are positive. It is now desirable that we seek similar conditions for the case of unrestricted $\tilde{r}_{j}$.

Suppose, first, that $-\infty \leqq \tilde{r}_{1}<0$ but $0<\tilde{r}_{2}<\infty$. By condition (4.22), when $z_{1}=\infty, z_{q+1}=\infty$ and therefore the point $z=\infty$ will be part of the locus $L_{12}$. (Cf. IV § 2.) As $\tilde{\omega}_{1}$ increases from $-\infty$, the quantities $\sigma_{12}$ and $\tau_{12}$ are interchanged. In any case, for large values of $-\tilde{\omega}_{1}$, the radius of circle $D_{12}$ will be

$$
-\left(1 /\left|m_{q+1}\right|\right)\left(\left|m_{2}\right| \tilde{\omega}_{2}+\left|m_{1}\right| \tilde{\omega}_{1}\right) .
$$

If, then, $\tilde{\omega}_{1}$ continues to increase to the value $-\left|m_{2} / m_{1}\right| \tilde{\omega}_{2}, D_{12}$ becomes a null circle and $L_{12}$ the entire plane. Briefly, if $r_{1} \leqq\left|m_{2} / m_{1}\right| r_{2}$, the locus $L_{12}$ is the entire plane; if $r_{1}>\left|m_{2} / m_{1}\right| r_{2}$, the locus $L_{12}$ is the exterior of the circle $D_{12}$ where $\Delta_{12}=\tau_{12}$ if $m_{1} m_{2}>0$ and $\Delta_{12}=\sigma_{12}$ if $m_{1} m_{2}<0$.

If both $\tilde{r}_{1}<0$ and $\tilde{r}_{2}<0$, the locus $L_{12}$ is always the entire plane. For, in allowing $\gamma_{1}$ and $\gamma_{2}$ to expand, we can choose $\tilde{\omega}_{1}$ as negative and $\tilde{\omega}_{2}$ as positive and so large that

$$
\tilde{\omega}_{1} \geqq-\left|m_{2} / m_{1}\right| \tilde{\omega}_{2} .
$$

In other words, if $\tilde{r}_{i}<0$ for any two $j$, the locus $R$ always contains the point at infinity.*

* The point $z_{q+1}$ defined by the equation (4.22) is the center of gravity $G$ of the other points $z_{j}$. When the first $q z_{j}$ vary over their regions $C_{j}$, the locus of $G$ will be, according to our methods,

(a) the entire plane, if, for at least two $k, \bar{r}_{k}<0$, or if, for at least one $j$ and one $k, \tilde{r}_{j}<0$ and

$$
\left|\bar{r}_{j}\right| \leqq\left|m_{k} / m_{j}\right|\left|\bar{r}_{k}\right| ;
$$

(b) the interior and circumference of the circle

if $\tilde{r}_{k}>0$ all $k$; or,

$$
B \equiv \sum_{\substack{i=1 \\ j-i+1}}^{q} m_{i} m_{j} \Delta_{i j}+m_{q+1} \sum_{1}^{q} m_{j} C_{j Q+1}=0
$$

(c) the exterior and circumference of the circle $H$ if $\tilde{r}_{k}<0$ for just one $k$. see BV, p. 60 .

The quantity $\Delta_{i j}$ is the same in $B$ as it is for the curve (4.42). For another derivation of (b), 
When $q=2$, an alternative method for deciding whether $z=\infty$ is part of the locus $R$ may be obtained directly. Equation (4.42) represents a circle which, formed for the $\boldsymbol{\gamma}_{j}$, is

$$
\Gamma=m_{1} m_{2} \Delta_{12} \gamma_{3}+m_{1} m_{3} \Delta_{13} \gamma_{2}+m_{2} m_{3} \Delta_{23} \gamma_{1}=0,
$$

with radius and center respectively

$$
\omega=\left|\frac{\nu}{\lambda}\right| \quad \text { and } \quad \alpha=\frac{\mu}{\lambda},
$$

where

$$
\begin{aligned}
& \lambda=m_{1} m_{2} \Delta_{12}+m_{1} m_{3} \Delta_{13}+m_{2} m_{3} \Delta_{23}, \\
& \mu=m_{1} m_{2} \Delta_{12} \alpha_{3}+m_{1} m_{3} \Delta_{13} \alpha_{2}+m_{2} m_{3} \Delta_{28} \alpha_{1}, \\
& \nu=m_{1} m_{2} \Delta_{12} \rho_{3}+m_{1} m_{3} \Delta_{13} \rho_{2}+m_{2} m_{3} \Delta_{23} \rho_{1} .
\end{aligned}
$$

Since, when the $\omega_{j}=0$, all $j, \Delta_{j k}>0$, and

$$
\lambda=-\left(\sum_{1}^{3} m_{j} z_{j}^{*}\right)\left(\sum_{1}^{3} m_{j} \bar{z}_{j}^{*}\right)<0,
$$

we always have $\tilde{\omega}=-\nu / \lambda$. Consequently, the locus $R$ is finite or not according as $\tilde{\omega}>0$ or $\tilde{\omega}<0$.

5. The entire plane as locus. To complete the study of the general case, we need yet to inquire into the circumstances under which $R$ is the entire plane.

As the regions $\gamma_{j}$ expand, one or more of the ovals of (4.32) may conceivably expand and sweep out the entire plane; or, after $R$ has acquired the point $z=\infty$, the oval bounding the part of $R$ containing $z=\infty$ may shrink to a point and its exterior thus become the entire plane. When $q=2$, these descriptive conditions are easily expressible in terms of $\omega$ and $\alpha$ of (4.43).

The locus $R$ may also become the entire plane discontinuously. If all the regions $C_{j}$ have a point in common, the instant the regions $\gamma_{i}$ are suffciently large all to include this common point does the equation $F=0$ become the identity

$$
\left(\frac{1}{z-z_{1}}\right) \sum_{1}^{q+1} m_{i}=0
$$

and therefore locus $R$ the entire plane. An example of this sort has been called to my attention by Professor Walsh. (See also B VII, p. 12.) If $q=2$, $m_{1}=m_{2}=1$, the regions $C_{1}$ and $C_{2}$ are the interior of the circle $C_{1} \equiv C_{2}$, and the region $C_{3}$ is merely the point at infinity, then $R$ is the locus of the zeros of 


$$
F=\frac{1}{z-z_{1}}+\frac{1}{z-z_{2}}
$$

or the locus of the point $z=\frac{1}{2}\left(z_{1}+z_{2}\right)$. Obviously $R$ is the interior of the circle $C=C_{1}$. If the region $C_{1}$ is suitably allowed to expand, the region $C$ remains coincident with it, until the region $C_{1}$ becomes a half-plane when, because all three regions $C_{j}$ have a common point, the region $C$ suddenly becomes the entire plane.

\section{Applications}

1. A cross-ratio theorem. We now return to the theorem which was the starting point of this paper (cf. I $\$ 1$ ), the theorem that "if the points $z_{1}, z_{2}, z_{3}$ vary independently and have circular regions as their respective loci, then the locus of the point $z$ defined by the equation

$$
\frac{m_{1}}{z-z_{1}}+\frac{m_{2}}{z-z_{2}}+\frac{m_{3}}{z-z_{3}}=0
$$

where $m_{1}, m_{2}, m_{3}$ are real constants whose sum is zero, is also a circular region."

By specializing throughout the preceding chapter the number $q$ to be two, we obtain at once (1) an analytical proof of this theorem; (2) conditions that the locus contain the point at infinity; (3) eonditions that the locus be the entire plane; and (4) the equation of the circle bounding the locus when the locus is not the entire plane. The latter equation is (cf. equation (4.42))

$$
m_{1} m_{2} \Delta_{12} C_{3}+m_{1} m_{3} \Delta_{13} C_{2}+m_{2} m_{3} \Delta_{23} C_{1}=0,
$$

where $\Delta_{j k}=\sigma_{j k}$ if $\left(m_{j} \tilde{r}_{j}\right)\left(m_{k} \tilde{r}_{k}\right)>0$ and $\Delta_{j k}=\tau_{j k}$ if $\left(m_{j} \tilde{r}_{j}\right)\left(m_{k} \tilde{r}_{k}\right)<0$.

As we remarked in the fifth footnote to chapter $I$, the above theorem may also be expressed in terms of a real constant cross ratio, instead of in terms of the partial fraction (5.1).

2. The jacobian of two binary forms. A second immediate application of the results of the preceding chapter is to the location of the zeros of the jacobian of the two binary forms

$$
f_{1}=\prod_{1}^{p_{1}}\left(u v_{j}-v u_{j}\right) \quad \text { and } \quad f_{2}=\prod_{p_{1}+1}^{p_{1}+p_{2}}\left(u v_{j}-v u_{j}\right) .
$$

By Euler's law for homogeneous functions, this jacobian $J_{12}$ may be written as

$$
J_{12}=\frac{f_{1} f_{2}}{v^{2}}\left\{\sum_{1}^{p_{1}} \frac{p_{2} v_{j} v}{u v_{j}-v u_{j}}+\sum_{p_{1}+1}^{p_{1}+p_{2}} \frac{\left(-p_{1}\right) v_{j} v}{u v_{j}-v u_{j}}\right\} .
$$

The zeros of $J_{12}$ are thus either multiple zeros of one lower order of the pro- 
duct $f_{1} f_{2}$, or zeros of the bracket in (5.21). (Cf. BI, p. 476.) On introduction of the non-homogeneous coördinates $z=u / v$, this bracket becomes the partial fraction

$$
F=\sum_{1}^{p_{1}} \frac{p_{2}}{z-z_{j}}+\sum_{p_{1}+1}^{p_{1}+p_{2}} \frac{\left(-p_{1}\right)}{z-z_{j}}
$$

As in III $\S 1$, we now obtain the following theorem:

If $f_{i j}, f_{1}=\Pi_{1}^{s} f_{1 i}$, and $f_{2}=\prod_{s+1}^{q+1} f_{2 j}$ are binary forms of degrees $n_{i j}, 1 / p_{1},-1 / p_{2}$ respectively, and if all the zeros of each $f_{i j}$ have as common locus a circular region $C_{j}$, the locus of the zeros of $J_{12}$ will consist of (a) the whole of every region $C_{i}$ for which $n_{i j} \neq 1$, and (b), when $q \geqq 2$, the ( $\left.q-1\right)$-circular domain specified in IV $\$ 4$ and $\$ 5$, where $m_{j}=p_{i} n_{i j}$.

Reasoning similar to that referred to in III $\$ 1$ will also establish the facts that there will exist in each region $C_{i}$ exactly $n_{i j}-1$ zeros of $J_{12}$ and $q-1$ in (b) if no two of the regions $C_{j}$ have any point in common and if, in addition, (b) does not overlap any region $C_{i}$; and precisely one zero in each part of (b) if furthermore (b) falls into $q-1$ distinct parts.

When $q=1$, the theorems of this section become identical with those of Bôcher (BI, p. 477) that, if all the zeros of $f_{1}$ and $f_{2}$ lie respectively in the circular regions $C_{1}$ and $C_{2}$, there are no zeros of $J_{12}$ outside of these two regions, and that, if the regions $C_{1}$ and $C_{2}$ do not overlap, there are exactly $p_{1}-1$ zeros of $J_{12}$ in the first and $p_{2}-1$ zeros of $J_{12}$ in the second region.

When $q=2$, the theorems of this section reduce to those due to Walsh (BIV, p. 112) that, if the circular regions $C_{1}, C_{2}, C_{3}$ are the respective loci of $m_{1}$ roots of $f_{1}$, the remaining $m_{2}$ roots of $f_{1}$, and all the $m_{3}$ roots of $f_{2}$, then the locus of zeros of $J_{12}$ will consist of the whole of each circular region $C_{i}$ for which $m_{j} \neq 1$ and the locus described in $\mathrm{V} \S 1$.

Similar theorems hold true for the covariant

of the forms

$$
\Phi=\frac{1}{k}\left(\prod_{1}^{k} f_{j}\right) \sum_{i, j=1}^{k} \frac{m_{i}}{p_{i}} \frac{J_{i j}}{f_{i} f_{j}}
$$

$$
f_{i}=\prod_{P_{j-1+1}}^{P_{j}}\left(u v_{i}-v u_{i}\right)
$$

where $P_{0}=0$, and $P_{j}-P_{j-1}=p_{j}$, the degree of $f_{i}$, and $\sum_{1}^{k} m_{i} p_{i}=0$. For, we may write

(See BI, p. 467.)

$$
\Phi=\frac{1}{v^{2}}\left(\prod_{1}^{k} f_{j}\right) \sum_{1}^{k} m_{j} \sum_{P_{j-1+1}}^{P_{j}} \frac{v v_{j}}{u v_{j}-v u_{j}}
$$


VI. The MOST General CASE

1. The locus $R$. Having obtained the locus $R$ in the special case II (the case of real numbers $m_{j}$ summing to zero but of arbitrary circular regions $C_{j}$ ) we are now in a position to study the locus $R$ in the case of unrestricted numbers $m_{j}$ and of unrestricted circular regions $C_{j}$. For, as we shall presently see, the results in the most general case can be deduced from those of special case II.

Let us consider the partial fraction

$$
F=\sum_{1}^{q+1} \frac{m_{j}}{z-z_{j}}+\frac{m_{q+2}}{z-z_{q+2}}
$$

where $n+m_{q+1}=0$ and $n=\sum_{1}^{q+1} m_{j}$. This fraction is of the type considered in the special case II, but, if the point $z_{q+2}$ is required to recede to infinity, the term in the subscript $q+2$ disappears from expression (6.11) and the fraction becomes one in which the sum of the $m_{j}$ 's is not necessarily zero.

In the particular instance of case II in which $q+2$ circular regions $C_{i}$ appear and the region $C_{q+2}$ consists merely of the point $z_{q+2}$, the locus $R$ is bounded by the curve (cf. equation (4.42))

$$
\sum_{j=1, k=j+1}^{q+1} \frac{m_{j} m_{k} \Delta_{j k}}{C_{j} C_{k}}+\sum_{1}^{q+1} \frac{m_{q+2} m_{j} C_{j q+2}}{P_{q+2} C_{j}}=0 .
$$

Let us now cause the point $z_{q+2}$ to move off to infinity. The above-considered locus $R$ will then become the locus $R$ of the unrestricted case. Since

$$
\frac{C_{j q+2}}{P_{q+2}}=\frac{x_{q+2}^{2}+y_{q+2}^{2}-2 a_{j} x_{q+2}-2 b_{j} y_{q+2}+c_{j}}{x_{q+2}^{2}+y_{q+2}^{2}-2 x x_{q+2}-2 y y_{q+2}+x^{2}+y^{2}}
$$

and

$$
\lim _{\mid z q+2}\left(\frac{C_{i q+2}}{P_{q+2}}\right)=1
$$

the locus $R$ of the general case will therefore be bounded by the curve

$$
\sum_{j=1, k=j+1}^{q+1} \frac{m_{j} m_{k} \Delta_{j k}}{C_{j} C_{k}}-\sum_{1}^{q+1} \frac{n m_{j}}{C_{j}}=0
$$

where $\Delta_{j k}=\sigma_{j k}$ if $\left(m_{j} \tilde{r}_{j}\right)\left(m_{k} \tilde{r}_{k}\right)>0$ and $\Delta_{j k}=\tau_{j k}$ if $\left(m_{k} \tilde{r}_{k}\right)\left(m_{k} \tilde{r}_{k}\right)<0$.

Thus we obtain a curve which clearly includes as special cases the curves (2.51) and (4.42) and which has, if $n \neq 0$, the same singular foci as curve (2.51).

In the same manner, we can carry over from the special case II all of 
the other important properties of the locus $R$. We see thereby that in every case the locus $R$ is a p-circular domain, where

$$
p=q-1+\lim _{\nu \rightarrow 0}\left(\frac{n}{n+\nu}\right) .
$$

2. Application to the derivative of a rational function. As a final application let us consider the location of the zeros of the derivative of the rational function

$$
f(z)=\frac{\prod_{1}^{u}\left(z-z_{j}\right)}{\prod_{u+1}^{u+v}\left(z-z_{j}\right)}
$$

where the numerator $z_{j}$ are distinct from the denominator ones. Since

$$
f^{\prime}(z)=f(z)\left\{\sum_{1}^{u} \frac{1}{z-z_{j}}-\sum_{u+1}^{u+v} \frac{1}{z-z_{j}}\right\},
$$

the zeros of $f^{\prime}(z)$ are the multiple zeros of $f(z)$ to one lower order, the point at infinity if $v-u>0$ and the zeros of the partial fraction in the brace. We may accordingly state the following theorem, which, when $q=1$ or 2, reduces to those proved by Walsh in BIV, pp. 114-115:

If $f_{1 j}(z), f_{2 i}(z), f_{1}=\Pi_{1}^{s} f_{1 j}$, and $f_{2}=\Pi_{s+1}^{q+1} f_{2 i}$ are polynomials of degrees $m_{j}$, $-m_{i}, u$ and $v$ respectively; if $f_{1}$ and $f_{2}$ have.no zeros in common, and if all the zeros of each polynomial $f_{t k}$ have as common locus a circular region $C_{k}$, then the locus of the zeros of the derivative of the rational function $f(z)=f_{1} / f_{2}$ will be

(a) the whole of each region $C_{j}$ for which $m_{j} \neq 1$ and $j=1,2,3, \cdots, s$;

(b) the point at infinity if $v-u>0$, and

(c) if $q \geqq 1$, the p-circular domain specified in the preceding section, VI $§ 1$.

\section{Generalizations}

1. Complex $m_{j}$. The methods of the preceding chapters permit us also to study the locus $R$ of the zeros of the partial fraction $F$ when the numbers $m_{i}$ are complex. ${ }^{*}$ We give here only the results in two special cases: case (a), where $q=1, m_{1}+m_{2} \neq 0$, and the circular regions $C_{i}$ are finite; and case (b), where $q=2, m_{1}+m_{2}+m_{3}=0$, and both the regions $C_{i}$ and the locus $R$ are finite.

* Cf. BVII for a geometrical discussion of cases of complex $m_{i}$, and BIX for a study of simple zero-free regions of the fraction $F$ in the case of complex $m_{i}$; also Walsh, these Transactions, vol. 24 (1922), pp. 163-180; p. 169. 
We shall in the statement of these results use the following additional notation:

$$
\begin{aligned}
\mu_{j k} & =\frac{1}{2}\left(\bar{m}_{j} m_{k}+\bar{m}_{k} m_{j}\right) ; \quad \nu_{j k}=\frac{i}{2}\left(\bar{m}_{j} m_{k}-m_{j} \bar{m}_{k}\right) ; \\
l_{z^{z} z_{k}} & =i\left(\bar{z}_{j} z_{k}-\ddot{z}_{k} z_{j}\right) ; \\
L_{j k} & =i\left\{\left(\bar{z}-\bar{\alpha}_{j}\right)\left(z-\alpha_{k}\right)-\left(z-\alpha_{j}\right)\left(\bar{z}-\bar{\alpha}_{k}\right)\right\} .
\end{aligned}
$$

In subcase (a), the locus $R$ is the interior and circumference of the circle $\left(\left|m_{1}\right|^{2}+\mu_{12}\right) C_{2}+\left(\left|m_{2}\right|^{2}+\mu_{12}\right) C_{1}$

$$
-\left\{\mu_{12} \sigma_{12}+2\left(\left|m_{1} m_{2}\right|-\mu_{12}\right) r_{1} r_{2}-\nu_{12} L_{12}\right\}=0,
$$

with center at the point

$$
\frac{1}{\left|m_{1}+m_{2}\right|^{2}}\left\{\left(\left|m_{1}\right|^{2}+\mu_{12}\right) \alpha_{2}+\left(\left|m_{2}\right|^{2}+\mu_{12}\right) \alpha_{1}\right\}
$$

and with a radius equal to

$$
\frac{\left|m_{1}\right| r_{2}+\left|m_{2}\right| r_{1}}{\left|m_{1}+m_{2}\right|}
$$

In subcase (b), the subcase of $\mathrm{V} \S 1$ for complex $\lambda$, the locus $R$ will be finite provided that the region $C_{3}$ does not overlap the interior or circumference of the circle

$$
\begin{aligned}
E=\mu_{28} C_{23}+\mu_{18} C_{13}+\mu_{12} \tau_{12}+2\left(\left|m_{1}\right|\left|m_{2}\right|\right. & \left.+\mu_{12}\right) r_{1} r_{2} \\
& -\nu_{23}\left(l_{\alpha_{1} \alpha_{2}}+l_{\alpha_{2} \alpha_{2}}+l_{z, \alpha_{1}}\right)=0,
\end{aligned}
$$

i.e., provided that. for all points $z_{3}$ in the region $C_{3}$,

$$
E\left(z_{3}\right)<0 \text {. }
$$

The locus $R$ then will consist of the interior and circumference of the circle

$$
\begin{aligned}
H=\left\{\mu_{12} \tau_{12}+\right. & \left.2\left(\left|m_{1}\right|\left|m_{2}\right|+\mu_{12}\right) r_{1} r_{2}+\nu_{12} L_{12}\right\} C_{3} \\
& +\left\{\mu_{31} \tau_{31}+2\left(\left|m_{3}\right|\left|m_{1}\right|+\mu_{31}\right) r_{3} r_{1}+\nu_{31} L_{31}\right\} C_{2} \\
& +\left\{\mu_{23} \tau_{23}+2\left(\left|m_{2}\right|\left|m_{3}\right|+\mu_{23}\right) r_{2} r_{3}+\nu_{23} L_{23}\right\} C_{3}=0 .
\end{aligned}
$$

In this equation the linear expressions $L_{j k}$ do not prevent $H=0$ from representing a circle.

As is easily seen, the results for both subcases agree with our previous ones if the $m_{j}$ are made real numbers.

2. Zeros of $F-\lambda$. Likewise our methods permit us to secure the locus of the zeros of $F-\lambda$, where $\lambda$ is an arbitrary constant, real or complex. As 
a sample of such results, we state the following theorem for the case of real positive $m_{j}$ and of finite regions $C_{j}$.

The locus of the zeros of $F-\lambda$ is the interior and the boundary of all the ovals of the $(q+1)$-circular $2(q+1)$-ic curve

$$
\lambda \bar{\lambda}-\sum_{1}^{q+1} \frac{m_{j} L_{j}}{C_{j}}+\sum_{1}^{q+1} \frac{n m_{j}}{C_{j}}-\sum_{j=1, k=j+1}^{q+1} \frac{m_{j} m_{k} \sigma_{j k}}{C_{j} C_{k}}=0,
$$

where $n=\sum_{1}^{q+1} m_{j}$, and

$$
L_{j}=\bar{\lambda}\left(\bar{z}-\bar{\alpha}_{j}\right)+\lambda\left(z-\alpha_{j}\right) .
$$

The singular foci of (6.21) are the zeros of $F-\lambda$ for $z_{j}=\alpha_{j}$.

As immediate applications of these results, we mention the location of the zeros of linear combinations of either a rational function and its derivative or the product of two binary forms and the jacobian of these forms.* For instance, the expression $f^{\prime}(z)-\lambda f(z)$, where $f(z)$ is a rational function and $\lambda$ an arbitrary constant, has its zeros at the multiple roots of $f(z)$ and at the zeros of $F-\lambda$, where $F$ is the partial fraction $f^{\prime} / f$.

In subsequent papers we hope to study further the properties of the locus $R$ and to give additional specializations and generalizations.

* Cf. Walsh, Bulletin of the American Mathematical Society, vol. 30 (1924), pp. 51-62, for a study of the zeros of linear combinations of a polynomial and $k$ of its derivatives.

HARVARD UNIVERSITY,

Cambridge, Mass. 